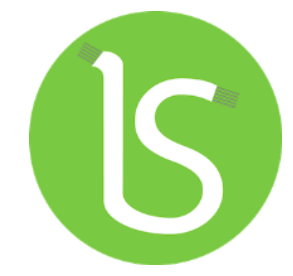

\title{
La microhistoria del cacao en Jalcomulco, Veracruz: participación social para un aprovechamiento sostenible desde la revalorización de saberes, recursos y capacidades locales
}

\section{David Omar Ayala Benítez a}

Resumen - La microhistoria del cacao en Jalcomulco, Veracruz; aborda las implicaciones sociohistóricas que se tejen a partir de este elemento como un ingrediente esencial en la preparación, del tradicional champurrado con cacao en la localidad. Esta bebida caliente, acompaña y se comparte en fechas especiales, adquiriendo un valor simbólico ligado a sus tradiciones. El cacao, se vincula a un consumo histórico local que envuelve saberes heredados y prácticas en torno a la forma en que se cultiva, procesa y prepara. De modo que la microhistoria, da cuenta de su origen en un principio, y expone sus relaciones: del contexto agrícola, prácticas entorno al cultivo, saberes heredados, participación por género, aprovechamiento, de los momentos y espacios de significación. Planteándose como base para una propuesta de aprovechamiento sostenible de cacao, que parte del reconocimiento y revalorización de saberes, recursos y capacidades locales para una gestión ambiental propia, orientada en la economía social.

Palabras clave - Aprovechamiento Sostenible, Cacao, Saberes, Jalcomulco, Recursos Locales.

Abstract - The microhistory of cacao in Jalcomulco, Veracruz; addresses the sociohistorical implications that are woven from this element as an essential ingredient in the preparation of the traditional champurrado with cocoa in the locality. This hot drink accompanies and is shared on special dates, acquiring a symbolic value linked to its traditions. Cocoa is linked to a local historical consumption that involves inherited knowledge and practices around the way it is grown, processed and prepared. So that microhistory, gives an account of its origin in the beginning, and exposes its relationships: of the agricultural context, practices around cultivation, inherited knowledge, participation by gender, use, of the moments and spaces of significance. Considering itself as the basis for a proposal for the sustainable use of cocoa, which starts from the recognition and revaluation of local knowledge, resources, and capacities for its own environmental management, oriented towards the social economy.

Keywords - Sustainable Use, Cocoa, Knowledge, Jalcomulco, Local Resources.

\section{CÓMO CITAR \\ HOW TO CITE:}

Ayala-Benítez, D. O. (202I). La microhistoria del cacao en Jalcomulco, Veracruz: participación social para un aprovechamiento sostenible desde la revalorización de saberes, recursos y capacidades locales. Interconectando Saberes, (I2), |85-|9|

https://doi.org//0.25009/is.v0il2 .2717

Recibido: 16 de junio de 2021 Aceptado: 16 de julio de 2021 Publicado: 20 de julio de 2021

${ }^{a}$ Universidad Veracruzana, México. E-mail: davidayalabeck@gmail.com 


\section{INTRODUCCIÓN}

El cultivo de cacao desde tiempos prehispánicos se concebía ligado a las selvas. Donde éste, se aprovechaba como parte de los componentes agrícolas asociados dentro de estos agroecosistemas. Los cuales, albergaban una compleja estructura y alta variedad de especies (Villavicencio, 2013, p.68). Este tipo de prácticas, se conservan en algunas regiones tropicales, replicándose con fines de reforestación, diversificación productiva, conservación y generación de corredores biológicos entre manchones de selva tropical (Ogata 2007, p.5).

A estos cultivos en asociación, que combinan espacial y temporalmente otros cultivos o componentes animales, dentro de la misma unidad de tierra, se les reconoce dentro de los sistemas agroforestales. Los cuales, se desarrollan sobre la base de optimizar los efectos benéficos de las interacciones entre sus componentes, con la intención de "obtener un patrón productivo que se compara con lo que generalmente se obtiene de los mismos recursos disponibles en un monocultivo" (Farrell y Altier, 1999).

Este manejo, en las selvas y cacao, tiene origen hace más de 3,500 años en Mesoamérica con la civilización Olmeca y al sur con la Maya. Existen también, vestigios de su consumo al menos desde hace 5 mil años en la alta Amazonía (Valdez, 20I3, p.2I). Esto, sugiere la domesticación y aprovechamiento de las selvas con cacao, de manera intensiva. Lo cual se hacía sin llegar a ser de manera extensiva o en monocultivo. Tomando en cuenta esquemas de diversificación desde una percepción de domesticación basada en organizar, conducir y diseñar; sin someter o condicionar la planta (Ogata, 2018).
Los sistemas y prácticas agroforestales también evitan el deterioro de la diversidad biológica, la deforestación y empobrecimiento del suelo. Así como, brindan la posibilidad de diversificar los productos que obtiene el agricultor. Su importancia radica, además, en su pertinencia actual para el uso integral y sostenible de los recursos naturales en diversos ecosistemas, como las selvas tropicales asociadas al cultivo de cacao.

Particularmente en la localidad de Jalcomulco en Veracruz, prevalece un arraigado consumo de cacao que refiere a un uso histórico como parte de los ingredientes esenciales para el champurrado tradicional, preparado en distintas fechas especiales. Existe también, un aprovechamiento incipiente por algunas familias que lo procesan de manera rústica y lo comercializan como chocolate artesanal.

Con incertidumbre sobre su llegada, agricultores de edad mayor dan cuenta de su existencia cerca de los "cañones" en lugares "frescos", creciendo a la sombra de árboles más altos. Si bien se mantiene entre la memoria de los habitantes, y con un consumo local actual, existe desconocimiento sobre su cultivo y beneficio. Ante esta situación, se plantea alentar, agrupar y promover una propuesta de aprovechamiento sostenible de cacao con base en la articulación de saberes, recursos y capacidades locales.

De esta manera, se busca fomentar formas de interacción alternativas a la explotación que deteriora el medio ambiente con prácticas y concepciones perjudiciales, incluso dinamizando el turismo alternativo hacía otros elementos como el campo. Se plantea al respecto, establecer una propuesta de aprovechamiento sostenible de cacao, a partir de promover la gestión de recursos bioculturales con base en el diálogo de saberes empíricos y conocimientos científicos. 
Jalcomulco es un lugar reconocido principalmente por localizarse al margen del río "Los pescados", donde se práctica el turismo de aventura desde hace más de tres décadas, siendo la segunda actividad económica más importante localmente. La primera, concierne a la actividad agrícola, con una superficie cultivada de $52.2 \mathrm{~km} 2$ de los $72.4 \mathrm{~km} 2$ que conforman el municipio, produciendo principalmente: mango, maíz y café (Secretaría de Finanzas y Planeación de Veracruz [SEFIPLAN], 2019) en monocultivo.

El objetivo de este proyecto se concreta definiéndose desde el enfoque del desarrollo sostenible, en sus tres ejes. A partir de una estrategia metodológica fundamentada en la recuperación de la microhistoria para cimentar las bases de la propuesta. La cual consistió en la definición de los actores claves involucrados, respecto de horizontes denominados "puntos de quiebre", asociados a distintas escalas de análisis. De esta forma dotando de sentido, temporalidad $y$ espacialidad los acontecimientos que develan la microhistoria del cacao en Jalcomulco.

Posteriormente se propone la caracterización morfológica de la variedad entorno a la viabilidad del cultivo en la zona; así como un programa de información, sensibilización y asesoría entorno al propósito general con base en el análisis previo. Se pretende así, transmitir las bases integrales a un grupo de participantes que a su vez repliquen y transmitan lo aprendido de manera sistemática a otros. Propiciando así, la autogestión de recursos naturales como el cacao para satisfacer: una demanda arraigada a un consumo tradicional, la posibilidad de comercialización de excedentes en grano, procesarlo como chocolate artesanal; así como vincularlo a la vocación turística del sitio.

\section{PROPUESTA}

El cacao, se vincula a un consumo histórico local que envuelve saberes heredados y prácticas en torno a la forma en que se cultiva, procesa y prepara. De modo que la microhistoria sirve para dar cuenta de su origen en un principio, y exponer sus relaciones: del contexto agrícola, prácticas entorno al cultivo, saberes heredados, participación por género, aprovechamiento, los momentos y espacios de significación.

Son diversas las implicaciones sociohistóricas, que se tejen a partir de este elemento como un ingrediente esencial en la preparación del tradicional champurrado con cacao en la localidad. Esta bebida caliente, acompaña y se comparte en fechas especiales, adquiriendo un valor simbólico ligado a sus tradiciones.

La microhistoria como la manera de acceder a este relato, lo hace por medio de la contrastación de diversas fuentes: registros visuales, escritos y orales; detallando lo micro de la escala local, a la par de denotar los hilos conductores que se tejen hacia lo macro. Se vale ésta de diversas herramientas que permiten la reconstrucción a partir de fuentes documentales, censales; y técnicas de investigación como la entrevista a profundidad y la observación participante; entre otras.

La recuperación micro histórica parte de la contrastación de las fuentes para en principio establecer el panorama temporal. Por medio de "puntos de quiebre" que precisan cambios imponentes, acontecimientos que influyen sobre ésta. Los cuáles se suscriben a circunstancias específicas de tiempo, modo y lugar con escalas de incidencia: local, regional, global. De esta manera se definió en la Figura I mediante una representación cronológica. 


\section{Figura I}

Línea del tiempo: "Puntos de quiebre micro históricos del cacao en Jalcomulco, Ver."

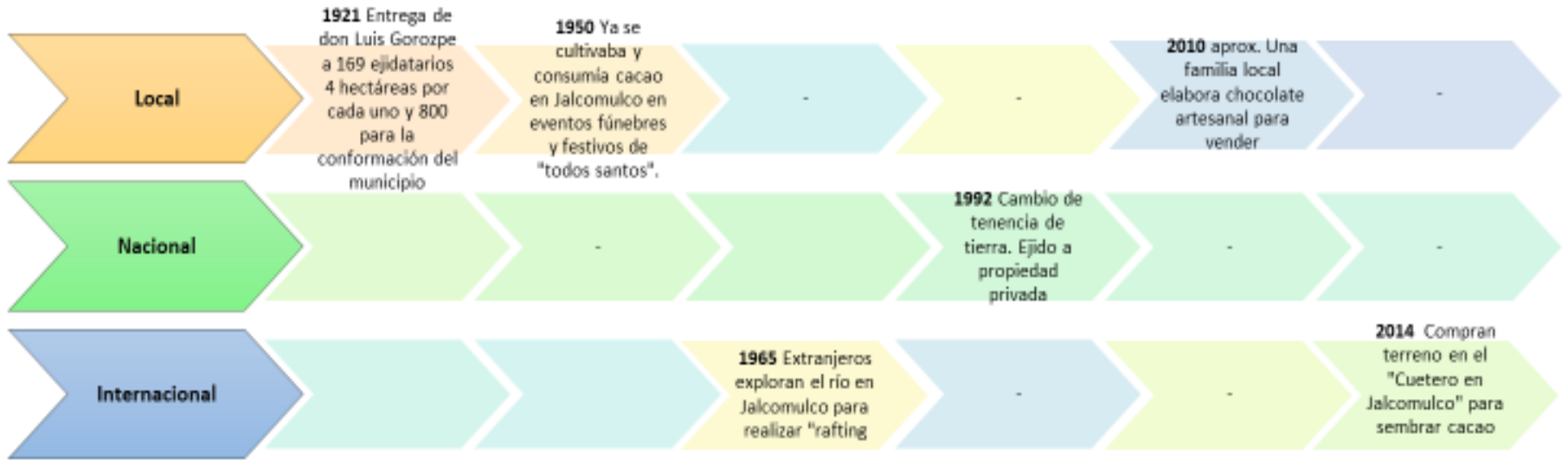

\section{Figura 2}

Indagación micro histórica

\section{Indagación micro-histórica}

\begin{tabular}{|} 
Pasado \\
1) Perfil - pasado: Adultos \\
mayores, hombres y/o mujeres, \\
que hayan vivido la mayor parte \\
de su vida en la localidad, \\
relacionados con la actividad \\
agrícola en Jalcomulco. Y, que \\
expresen conocer sobre la \\
historia del cacao localmente
\end{tabular}

\begin{tabular}{|l} 
Pr \\
\hline pre \\
act \\
pro \\
\hline
\end{tabular}

\section{Presente}

2) Perfil - presente: Personas que sean parte de la comunidad, que actualmente estén cultivando o procesando cacao en Jalcomulco como una actividad económica ya sea principal o complementaria para el ingreso familiar.

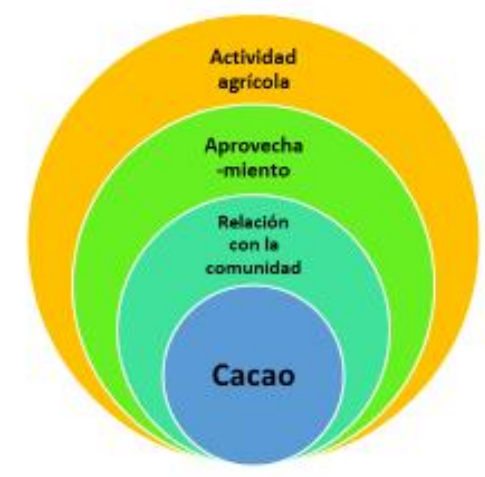

Perfiles de informantes clave 


\section{Figura 3}

Diagrama de ven: aprovechamiento sostenible de cacao
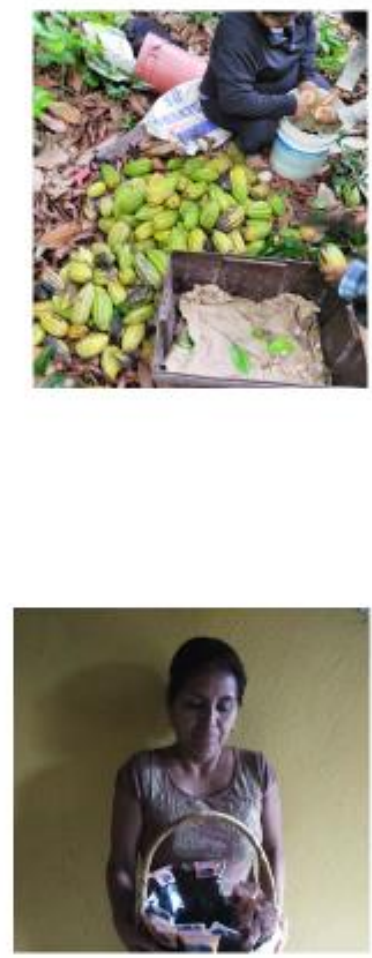

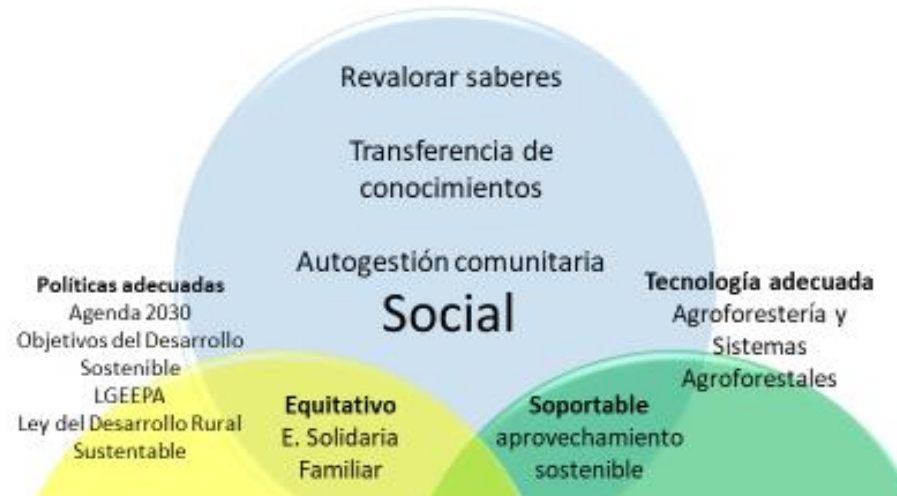

\section{Económico}

Diversificación productiva

Ahorro en agroquimicos

Comercialización de productos
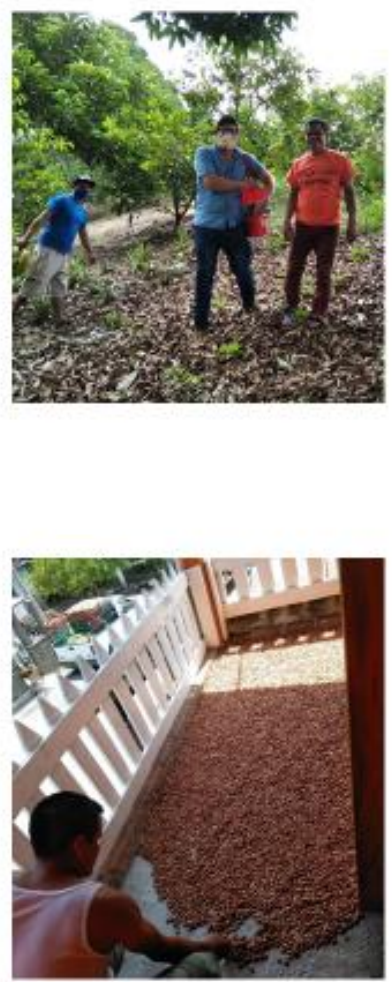

Con base en la Figura 2, se continuó con el trazo de perfiles de los informantes clave. Se estableció ciertas correlaciones como: horizontes temporales a cubrir (pasado y presente), así como su correlación con ejes de investigación que estructuraron el diseño del cuestionario para la aplicación de entrevistas a profundidad orientadas a indagar sobre la microhistoria del cacao en Jalcomulco.

La Figura 3, implicó la definición de ejes de análisis reconstruidos con base en las declaraciones expresadas por los actores y fuentes consultadas: actividad agrícola, aprovechamiento, relación con la comunidad, cacao. Los cuales a su vez permitieron justamente a través de éstos, delinear la historia local y disgregar sus elementos para mirarlos desde la perspectiva de la sostenibilidad.
Así fueron emergiendo las conceptualizaciones, "formas de pensar" y "de hacer", sobre: cultivo, chocolate, champurrado; y las representaciones que las sustentan. Reconstruyéndose a partir de ello las prácticas, significaciones y valoraciones respecto del cacao en la localidad, como punto de partida para plantearse un aprovechamiento sostenible con base en la articulación de los recursos, capacidades y saberes locales.

Es decir: "encontrar lo específico de cada sociedad y comprender las acciones de la gente en su contexto y en su tiempo" (Arias, 2006, p.186). Entendiéndose los grupos sociales como abiertos y expuestos a influencias que en la comprensión de sus particularidades exhiben las diferencias que permiten correspondencias más amplías. 
"Lo importante no es el tamaño de la sede donde se desarrolla sino la pequeñez y cohesión del grupo que se estudia, lo minúsculo de las cosas que se cuentan de él y la miopía con que se les enfoca" (González, 20 I I, p.I I).

Se atiende desde esta perspectiva, el examen de las prácticas y sus significaciones dentro de la organización y dinámicas sociales para el entendimiento de la relación naturaleza - sociedad. Se reconoce la microhistoria como una vía metodológica para expresar la complejidad de los procesos y sus estructuras. Se observa en ésta su pertinencia epistemológica para abordar el estudio de la complejidad en las relaciones, la representación a partir de lo "minúsculo" como insumo para la toma de decisiones (Fabre et al, 20I7, p.29). Y la capacidad de ésta para abordar desde perspectivas integrales, posibilitando abonar hacia otros campos como la elaboración de políticas públicas o la gestión adecuada de recursos bioculturales como el cacao.

\section{CONCLUSIÓN}

Planteándose como base para una propuesta de aprovechamiento sostenible de cacao, que parte del reconocimiento y revalorización de saberes, recursos y capacidades locales para una gestión ambiental propia, orientada en la economía social; la microhistoria permite desglosar con un orden temporal y espacial los hechos que la componen para analizarlos individualmente o en su conjunto.

Permitió a través de ella dar cuenta de prácticas, significaciones y las formas de socialización entorno al cacao en Jalcomulco, Veracruz. De tal forma, se concretó el champurrado tradicional de cacao como el símbolo de un arraigado consumo de este grano a través esta bebida. La cual, es propia de ciertas fechas especiales como fiestas de "todos santos", en eventos funerarios y épocas decembrinas.

Así mismo, sin determinar su origen se estableció con un referente de al menos 65 años atrás en la localidad, su importancia a través de personas que incluso vendían sus excedentes. Sobre las prácticas de cultivo, este persiste en la memoria de quienes se dedican al campo, como un árbol que crece en las laderas de los cañones, donde recibe sombra regular y el ambiente es fresco.

Existen árboles "madre", de donde actualmente se han reproducido árboles por semilla con la intención de conservar la variedad local de características criollas. Se han establecido cerca de riachuelos, a la sombra de árboles que le proporcionen sombra, en una unidad compuesta por diversos cultivos. Sin embargo, es necesario reforzar la importancia desde el enfoque de la agroforestería.

Por otra parte, tras la cosecha el proceso de beneficiado practicado consiste en desgranar la mazorca para lavar las semillas y exponerla posteriormente al sol. A esta forma de beneficiar el cacao, se le conoce como "Lavado y secado", en la cual no existe un cuidado sobre la fermentación de este a diferencia del cacao fermentado. Cada uno, acentúa ciertas características acorde a sus particularidades. El cacao en Jalcomulco se utiliza fundamentalmente para el champurrado, elaborando barras de chocolate rústico que se añade al gusto.

Este tipo de chocolate se hace de manera artesanal, se realiza la molienda de los granos, agregando azúcar y canela por lo general. El refinamiento de molienda, conchado, así como la separación de la pasta 
de cacao, se identificaron como pasos que permitirían mejorar los productos.

Hasta aquí se recuperó los diversos elementos que integran la microhistoria del cacao en Jalcomulco, Veracruz en relación con los principales hechos y actores que están involucrados. La perspectiva de análisis desde el desarrollo sostenible permitió disgregar los elementos de esta en sus tres dimensiones principales hacia el diseño de una propuesta que en este sentido permita la revalorización de los recursos bioculturales que se encuentran localmente.

Se pretende abonar a la utilización del cacao desde la gestión ambiental propia para la práctica de su cultivo asociado a una agricultura sostenible $y$ a sistemas agroforestales. Los cuales a su vez relacionan diversos componentes brindando la posibilidad de obtener diversos productos de acuerdo con los ciclos anuales $y$ el espacio, así como un manejo integrado del ecosistema con el fin de disminuir su acelerada degradación.

Por otra parte, se propone el cultivo de cacao para el abastecimiento de un consumo local, con la posibilidad de comercializar sus excedentes. E incluso vinculándolo a dinámicas como el agroturismo, de acuerdo con la vocación de sitio ligada al turismo alternativo. A través del cual puede generarse un ingreso con base en la preservación de agroecosistemas, prácticas sostenibles y con la posibilidad de difundir esta perspectiva a otros.

\section{REFERENCIAS}

Arias, P. (2006). Luis González: Microhistoria e historia regional. Desacatos, (21), 177-186.

http://www.scielo.org.mx/scielo.php?script=sci_a rttext\&pid=S1607-

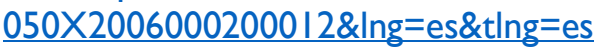

Farrell, J. G., y Altieri, M. A. (1999). Sistemas agroforestales. En Altieri, M. A. (ed), Agroecología. Bases científicas para una agricultura sustentable.

González, L. (20I I). Otra invitación a la microhistoria. Fondo de Cultura Económica.

Ogata, N. (2007). El cacao. Biodiversitas, 72(3), 2-5.

Ogata, N. (2018). El cacao como sistema de diversificación productiva para el desarrollo del sureste de México. En: Castillo, G. (Ed.). Cacao: alimento divino. Fundación Herdez. 59-82.

Fabre-Platas, D. A., Egea-Jiménez, C. y Caracas-Lozada, A. (20I7). Vías de acercamiento a los rostros de Andalucía a través de sus ciudades. En La microhistoria y lo urbano: Conocer, sentir, vivir las ciudades andaluzas (pp. 23-43). Editorial Universidad de Granada.

Secretaría de Finanzas y Planeación de Veracruz [SEFIPLAN]. (2019). Cuadernillos municipales 2019: Jalcomulco. Veracruz, México. Centro Estatal de Información Estadística y Geográfica de Veracruz. Gobierno del Estado de Veracruz.

Valdez, F. (20I3). Mayo Chinchipe. Hacia un replanteamiento del origen de las sociedades complejas en la civilización andina. En Valdez, F. (Ed). Arqueología Amazónica. Las civilizaciones ocultas del bosque tropical.

Villavicencio-Enríquez, L. (20I3). Caracterización agroforestal en sistemas de café tradicional y rústico, en San Miguel, Veracruz, México. Revista Chapingo. Serie ciencias forestales y del ambiente, 19(I), 67-80. 